\title{
The local ill-posedness of the modified KdV equation
}

\author{
by
}

\author{
Björn BIRNIR ${ }^{*}$, Gustavo PONCE ${ }^{*}$ and Nils SVANSTEDT ${ }^{\dagger}$ \\ Department of Mathematics, \\ University of California, \\ Santa Barbara, CA 93106.
}

\begin{abstract}
We find a new method for proving the local ill-posedness of the Cauchy problem for non-linear partial differential equations. The method is used to prove that the Cauchy problem for the Modified $\mathrm{KdV}$ equation is ill-posed in Sobolev spaces $H^{s}(R), s<-1 / 2$.

Key words: Initial value problem, well-posed, $\mathrm{KdV}$.

RÉSUMÉ. - Nous présentons une nouvelle méthode pour démontrer que le problème de Cauchy est localement mal posé dans des équations aux dérivées partielles non linéaires. Nous appliquons cette méthode pour démontrer que le problème de Cauchy pour l'équation de $\mathrm{KdV}$ modifiée est mal posé dans l'espace de Sobolev $H^{s}(R), s<-1 / 2$.
\end{abstract}

\section{INTRODUCTION}

Consider the initial value problem (IVP) of the Modified KdV equation

$$
\begin{aligned}
& \frac{\partial u}{\partial t}+\frac{\partial^{3} u}{\partial x^{3}}+u^{2} \frac{\partial u}{\partial x}=0 \quad t, x \in \mathbb{R}, \\
& u(x, 0)=u_{0}(x) .
\end{aligned}
$$

\footnotetext{
* Partially supported by the National Science Foundations grants nos. DMS-91-04532 and DMS-93-01351.

† Supported by grants from the Swedish Natural Science Research Council and NUTEK. 
It was proven in [6] that this IVP is locally well-posed in Sobolov spaces, $H^{s}(\mathbb{R})=(1-\Delta)^{-s / 2} L^{2}(\mathbb{R})$, for $s \geq 1 / 4$, and in addition that the IVP has a global solution in $H^{s}, s \geq 1$. Local well-posedness means that there exists a unique solution in $H^{s}$ for a small time interval $[0, \mathrm{~T}]$, it is a continous curve in $H^{s}$, originating in $u_{0}$ and the solution depends continously upon the data.

In this paper we introduce a technique to prove ill-posedness and apply it to the IVP (1.1). A known technique for showing ill-posedness uses a finite-time blow-up of the solution to the IVP, see [4]. If the solution and its lifespan $T^{*}$ scales with a parameter $\lambda$ then one can scale the solution to get arbitrarily small lifespans $T^{*} / \lambda$, as $\lambda \rightarrow \infty$. This contradicts the existence of a non-zero time interval $[0, \mathrm{~T}]$ where the solution exists. Our technique is very different from this but it seems to be of general use. In a subsequent publication [2] it will be applied to prove the ill-posedness of the generalized KdV equations, see [3], [6] and (1.2) below, and nonlinear Schrödinger equations in any dimension. The idea is to show that the solution, of (1.1), does not depend continously on its data in $H^{s}, s<-1 / 2$, by constructing a sequence converging (strongly) to the data in $H^{s}$ and then showing that the corresponding sequence of solutions does not converge (strongly) in $H^{s}$.

The sequence consists of the solitary wave solutions of (1.1), see [5] and [7], and the data is chosen to be the Dirac delta function. Thus we show that the IVP of the generalized $\mathrm{KdV}$ equations,

$$
\begin{aligned}
& \frac{\partial u}{\partial t}+\frac{\partial^{3} u}{\partial x^{3}}+u^{n} \frac{\partial u}{\partial x}=0 \quad t, x \in \mathbb{R}, \quad n \in \mathbb{Z}^{+}, \\
& u(x, 0)=\delta(x)
\end{aligned}
$$

is locally ill-posed in $H^{s}, s<-1 / 2$, for $n=2$. In [6] (1.2) was shown to be locally well-posed in $H^{s}, s \geq-3 / 4$, for $n=1$, and in [2] (1.2) will be shown to be locally ill-posed in $H^{s_{n}}, s_{n}<1 / 2-2 / n$, for $n \geq 3$, compare [8]. The Dirac delta function lies in $H^{s}, s<-1 / 2$, this is sufficiently smooth initial data for $\mathrm{KdV}$, borderline for $\mathrm{MKdV}$ and too rough for the (higher order) generalized $\mathrm{KdV}$ equations. 


\section{SOLITARY WAVES}

Consider the IVP

$$
\begin{aligned}
& \frac{\partial u}{\partial t}+\frac{\partial^{3} u}{\partial x^{3}}+u^{n} \frac{\partial u}{\partial x}=0 \quad t, x \in \mathbb{R}, \quad n \in \mathbb{Z}^{+}, \\
& u(x, 0)=u_{0}(x) .
\end{aligned}
$$

For any $n$ there exists a one-parameter $(k)$ family of solutions

$$
u_{n}(x, t, k)=\left(\frac{n+2}{2}\right)^{\frac{1}{n}} k^{\frac{2}{n}} \operatorname{sech}^{\frac{2}{n}}\left(\frac{n}{2}\left(k x-k^{3} t\right)\right), \quad 0<k<\infty,
$$

which are solitary waves.

The norm of these solutions is finite, with the exception of $\mathrm{KdV}(n=1)$, in the Sobolev spaces $H^{\frac{1}{2}-\frac{2}{n}}$, and independent of $k$, as $k \rightarrow \infty$. This is easily shown by use of the Fourier transform,

$$
\hat{u}_{n}(\xi, 0)=\frac{(2 n+2)^{1 / n}}{n \Gamma(2 / n)} \frac{1}{k^{1-\frac{2}{n}}} \Gamma\left(\frac{1}{n}+\frac{i \xi}{n k}\right) \Gamma\left(\frac{1}{n}-\frac{i \xi}{n k}\right),
$$

where $\Gamma$ is the gamma function, see Batemann [1]. The Fourier transform reduces to

$$
\hat{u}_{1}(\xi, 0)=\frac{3 \pi}{2} \xi \operatorname{csch}\left(\frac{\pi \xi}{2 k}\right)
$$

and

$$
\hat{u}_{2}(\xi, 0)=\sqrt{2} \pi \operatorname{sech}\left(\frac{\pi \xi}{2 k}\right)
$$

for $n=1$ and 2 , respectively.

LEMMA 2.1. - The solitary wave solution $u_{n}(x, t, k)$ has a finite $H^{s}$ norm, $s=\frac{1}{2}-\frac{2}{n}$, uniformly with respect to $k>0$, for $n>2$.

Proof. - By the Plancherel identity,

$$
\begin{aligned}
& \|u\|_{\frac{1}{2}-\frac{2}{n}}^{2}=\frac{(2 n+4)^{2 / n}}{4 \pi^{2}} \frac{1}{n^{2} \Gamma^{2}\left(\frac{2}{n}\right)} \frac{1}{k^{2-\frac{4}{n}}} \\
& \quad \times \int_{-\infty}^{\infty}\left(1+\xi^{2}\right)^{\frac{1}{2}-\frac{2}{n}} \Gamma^{2}\left(\frac{1}{n}+\frac{i \xi}{k n}\right) \Gamma^{2}\left(\frac{1}{n}-\frac{i \xi}{k n}\right) d \xi \\
& =(2 n+4)^{2 / n} \frac{1}{n^{2} \Gamma^{2}\left(\frac{2}{n}\right)} \int_{-\infty}^{\infty}\left(\frac{1}{k^{2}}+z^{2}\right)^{\frac{1}{2}-\frac{2}{n}} \\
& \quad \times \Gamma^{2}\left(\frac{1}{n}+\frac{i z}{n}\right) \Gamma^{2}\left(\frac{1}{n}-\frac{i z}{n}\right) d z
\end{aligned}
$$

Vol. 13, n 4-1996. 
where $z=\xi / k$. Then we use Stirling's formula to obtain

$$
\|u\|_{\frac{1}{2}-\frac{2}{n}}^{2}=\frac{c(n, k)(2 n+4)^{2 / n}}{n^{2} \Gamma^{2}\left(\frac{2}{n}\right)} \int_{-\infty}^{\infty} \frac{\left(z^{2}+\frac{1}{k^{2}}\right)^{\frac{1}{2}-\frac{2}{n}} e^{-\frac{2 \pi|z|}{n}}}{\left(\left(1-\frac{1}{n}\right)^{2}+\frac{z^{2}}{n}\right)^{1-\frac{2}{n}}} d z
$$

where $c(n, k)$ is a regular function of $k$. The last integral converges uniformly in $k$ and by the dominated convergence theorem we can pass to the limit $k \rightarrow \infty$, to get

$$
\left\|u_{n}(x, 0, \infty)\right\|_{\frac{1}{2}-\frac{2}{n}}=\frac{c(n)(2 n+4)^{2 / n}}{n^{2} \Gamma^{2}\left(\frac{2}{n}\right)} \int_{-\infty}^{\infty} \frac{z^{1-\frac{4}{n}} e^{-\frac{2 \pi|z|}{n}}}{\left(\left(1-\frac{1}{n}\right)^{2}+\frac{z^{2}}{n}\right)^{1-\frac{2}{n}}} d z .
$$

This integral converges (at the origin) only if $n>2$.

Remark 2.1. - The limit $k \rightarrow \infty$ gives the norm

$$
\|u\|_{s}=\left(\int_{-\infty}^{\infty}\left[(-\Delta)^{\frac{s}{2}} u\right]^{2} d x\right)^{1 / 2}
$$

of the homogeneous Sobolev space $\dot{H}^{s}$. This norm $\|\dot{u}\|_{\frac{1}{2}-\frac{2}{n}}$ is actually independent of $k$. Therefore the index $s=\frac{1}{2}-\frac{2}{n}$ is called the scaling index for $n$.

Remark 2.2. - Observe that if $u(x, t)$ solves (2.1), so does $\lambda^{2 / n} u\left(\lambda x, \lambda^{3} t\right)$ for any real-valued $\lambda>0$. This suggests that one should consider the homogeneous norm $\|\dot{u}\|_{\frac{1}{2}-\frac{2}{n}}$, and this agrees with [6] for $n \geq 4$. However, for $n=1$ and 2 the formulas (2.4) and (2.5) easily show that the $\dot{H}^{s}$ norms of $u_{n}(x, t, k)$ diverge as $k \rightarrow \infty$, for $s<-3 / 2$ and $s<-1 / 2$, respectively. One must consider the usual inhomogeneous $H^{s}$ norms to overcome this difficulty.

\section{THE MODIFIED KdV EQUATION}

Consider the IVP for the modified $\mathrm{KdV}$ equation (MKdV)

$$
\begin{aligned}
& \frac{\partial u}{\partial t}+\frac{\partial^{3} u}{\partial x^{3}}+u^{2} \frac{\partial u}{\partial x}=0, \quad x, t, \in \mathbb{R}, \\
& u(x, 0)=u_{0}(x) .
\end{aligned}
$$


We will show that the solution of this IVP cannot depend continuously on its initial data in a Sobolev space $H^{s}$ of negative index $s<-\frac{1}{2}$. The soliton solution (2.2) of MKdV scales to a constant multiple of the Dirac delta function,

$$
\lim _{\epsilon \rightarrow 0} \frac{\sqrt{2}}{\epsilon} \operatorname{sech}\left(\frac{x}{\epsilon}\right)=c \delta(x),
$$

this is proven in two lemmas below, and we will pose the IVP with this initial data in $H^{s}, s<-1 / 2$, to prove the theorem.

LEMMA 3.1. - The $H^{s}$ norm of

$$
u_{\epsilon}(x, 0)=\frac{\sqrt{2}}{\epsilon} \operatorname{sech}\left(\frac{x}{\epsilon}\right)
$$

is finite for $s<-1 / 2$ and

$$
\lim _{\epsilon \rightarrow 0}\left\|u_{\epsilon}(x, 0)\right\|_{s}=\|\sqrt{2} \pi \delta(x)\|_{s}
$$

for $s<-1 / 2$.

Proof. - The Fourier transform of $u_{\epsilon}$, see (2.5), is

$$
\begin{aligned}
\hat{u}_{\epsilon}(\xi, 0) & =\frac{\sqrt{2}}{\epsilon} \int_{-\infty}^{\infty} \operatorname{sech}\left(\frac{x}{\epsilon}\right) e^{-i \xi x} d x \\
& =\sqrt{2} \pi \operatorname{sech}\left(\frac{\epsilon \pi \xi}{2}\right) .
\end{aligned}
$$

By the Plancherel identity

$$
\begin{aligned}
\left\|u_{\epsilon}\right\|_{-s}^{2} & =\frac{1}{2} \int_{-\infty}^{\infty} \frac{\operatorname{sech}^{2}(\epsilon \pi \xi / 2)}{\left(1+\xi^{2}\right)^{s}} d \xi \\
& \leq \frac{1}{2} \int_{-\infty}^{\infty} \frac{1}{\left(1+\xi^{2}\right)^{s}} d \xi<\infty
\end{aligned}
$$

for $s>1 / 2$. Thus the integral converges uniformly in $\epsilon$ and we can bring the limit inside it. Finally one observes that

$$
\|\delta\|_{-s}^{2}=\frac{1}{2 \pi^{2}} \cdot \frac{1}{2} \int_{-\infty}^{\infty} \frac{1}{\left(1+\xi^{2}\right)^{s}} d \xi
$$

by the Plancherel identity, since $\hat{\delta}=1$.

Vol. 13, n 4-1996. 
Remark 3.1. - The integrals are invariant with respect to translation and this implies that we get the same statements for $u_{\epsilon}(x, t)$,

$$
\left\|u_{\epsilon}(x, t)\right\|_{s}<\infty
$$

and

$$
\lim _{\epsilon \rightarrow 0}\left\|u_{\epsilon}(x, t)\right\|_{s}=\|\sqrt{2} \pi \delta\|_{s}
$$

for $t \geq 0$ and $s<-1 / 2$.

LEMMA 3.2. - $u_{\epsilon}(x, 0)$ converges weakly to $\sqrt{2} \pi \delta(x)$, as $\epsilon \rightarrow 0$, but $u_{\epsilon}(x, t)$ converges weakly to zero, for $t>0$, as $\epsilon \rightarrow 0$, in $H^{s}, s<-1 / 2$.

Proof. - The $H^{-s}$ norms are finite

$$
\left\|u_{\epsilon}(x, 0)\right\|_{-s}=\left\|u_{\epsilon}(x, t)\right\|_{-s}<\infty
$$

by Lemma 3.1 and Remark 3.1, so it suffices to compute the limits

$$
\lim _{\epsilon \rightarrow 0}\left\langle u_{\epsilon}, v\right\rangle
$$

for all $v$ in a strongly dense subset of the dual space $H^{s}, s>1 / 2$, where $\langle$,$\rangle denotes the dual pairing between H^{-s}$ and $H^{s}$. We choose the smooth compactly supported functions $C_{0}^{\infty}$, they are dense in $H^{s}, s>0$. Then

$$
\begin{aligned}
\lim _{\epsilon \rightarrow 0} & \sqrt{2} \int_{-\infty}^{\infty} \frac{\operatorname{sech}}{\epsilon}\left(\frac{x}{\epsilon}\right) \phi(x) d x \\
& =\lim _{\epsilon \rightarrow 0} \sqrt{2} \int_{-\infty}^{\infty} \operatorname{sech}(y) \phi(\epsilon y) d y \\
& =\sqrt{2} \pi \phi(0), \quad \text { for } \phi \in C_{0}^{\infty},
\end{aligned}
$$

by the uniform convergence of the integral. This shows that $\frac{\sqrt{2}}{\epsilon} \operatorname{sech}\left(\frac{x}{\epsilon}\right)$ converges weakly to $\sqrt{2} \pi \delta(x)$, in $H^{-s}$. Similarly

$$
\begin{aligned}
& \lim _{\epsilon \rightarrow 0} \int_{-\infty}^{\infty} \frac{\operatorname{sech}}{\epsilon}\left(\frac{x}{\epsilon}-\frac{t}{\epsilon^{3}}\right) \phi(x) d x \\
& =\lim _{\epsilon \rightarrow 0} \int_{-\infty}^{\infty} \operatorname{sech}(y) \phi\left(\epsilon y+\frac{t}{\epsilon^{2}}\right) d y=0,
\end{aligned}
$$

since $\phi \in C_{0}^{\infty}$ has compact support. This shows that $\frac{\sqrt{2}}{\epsilon} \operatorname{sech}\left(\frac{x}{\epsilon}-\frac{t}{\epsilon^{3}}\right)$ converges weakly to 0 in $H^{-s}$.

We can now prove the result. 
THEOREM 3.1. - The initial value problem (3.1) for the modified $K d V$ equation is locally ill-posed in $H^{s}, s<-1 / 2$.

Proof. - We will prove that if there exists a local (strong) solution of the IVP (3.1) with $u_{0}(x)=\sqrt{2} \pi \delta(x)$ in $H^{s}, s<-1 / 2$, then it does not depend continuously on its initial data.

The $H^{s}$ norm of $u_{\epsilon}(x, 0)$ converges to the norm of $\sqrt{2} \pi \delta(x)$ by Lemma 3.1 and $u_{\epsilon}(x, 0)$ converges weakly to $\sqrt{2} \pi \delta(x)$ by Lemma 3.2 , therefore $u_{\epsilon}(x, 0)$ converges strongly to $\sqrt{2} \pi \delta(x)$. Now we solve the IVP (3.1) with initial data

$$
u_{0}(x, \epsilon)=\frac{\sqrt{2}}{\epsilon} \operatorname{sech}\left(\frac{x}{\epsilon}\right)
$$

to get the explicit solutions

$$
u_{\epsilon}(x, t)=\frac{\sqrt{2}}{\epsilon} \operatorname{sech}\left(\frac{x}{\epsilon}-\frac{t}{\epsilon^{3}}\right) .
$$

By Remark 3.1, the $H^{s}$ norm of $u_{\epsilon}(x, t)$ converges to the norm of $\sqrt{2} \pi \delta(x)$, which is non-vanishing but, by Lemma 3.2, $u_{\epsilon}(x, t)$ converges weakly to zero. Consequently, $u_{\epsilon}(x, t)$ cannot converge strongly to the solution of the IVP (3.1) with initial data

$$
u_{0}(x)=\sqrt{2} \pi \delta(x)
$$

in $H^{s}, s<-1 / 2$.

\section{REFERENCES}

[1] Batemann Manuscript Project, Tables of Integral Transforms, Vol. I Mc Graw-Hill, New York, 1954.

[2] B. Birnir, Kenig C. E., Ponce G., Svanstead N. and Vega L., On the ill-posedness of the IVP for the generalized Korteweg-de Vries and nonlinear Schrödinger equations, to appear in the Journal of the London Math. Soc., 1996.

[3] C. S. Gardner, Korteweg de Vries equation and generalizations IV. The Korteweg de Vries equation as a Hamiltonian system, J. Math. Phys., Vol. 12, 1971, pp. 1548-1551.

[4] R. T. Glassey, On the blowing-up solutions to the Cauchy problem for nonlinear Schrödinger equations, J. Math. Phys., Vol. 18, 1979, pp. 1794-1797.

[5] C. S. Gardner, T. H. Greene, M. D. Kruskal and R. M. Miura, Methods for solving the Korteweg-de Vries equation, Physical Review Letters, Vol. 19, 1967, pp. 1095-1097.

[6] C. E. Kenig, G. Ponce and L. Vega, Well-posedness and scattering results for the generalized Korteweg-de Vries equation via contraction principle, Comm. Pure Appl. Math., Vol. 46, 1993, pp. 527-620.

[7] R. M. Miura, Korteweg-de Vries equation and generalizations I. A remarkable explicit nonlinear transformation, J. Math. Phys., Vol. 9, 1968, pp. 1202-1204.

[8] J. Rauch, Nonlinear superposition and absorption of delta waves in one space dimension, J. Funct. Anal., Vol. 73, 1987, pp. 152-178. 